\title{
miR-205 promotes the growth, metastasis and chemoresistance of NSCLC cells by targeting PTEN
}

\author{
LIN LEI $^{1}$, YAPING HUANG ${ }^{2}$ and WENRONG GONG ${ }^{3}$ \\ Departments of ${ }^{1}$ Oncology and ${ }^{2}$ Respiratory Medicine, Xiangyang Central Hospital, Xiangyang 441021; \\ ${ }^{3}$ Institute of Oncology, Medical College of Hubei University of Arts and Science, Xiangyang, Hubei 441053, P.R. China
}

Received June 25, 2013; Accepted August 2, 2013

DOI: 10.3892/or.2013.2755

\begin{abstract}
Non-small cell lung cancer (NSCLC) is one of the most common causes of cancer-related mortality worldwide. microRNAs (miRNAs) play critical roles in carcinogenesis. miR-205 has been shown to be upregulated in NSCLC. In the present study, we identified the promotive effects of miR-205 on various significant biological properties of NSCLC cells, and confirmed the regulation of PTEN by miR-205. The expression of miR-205 was examined by quantitative real-time PCR both in NSCLC cell lines and tissues. The effect of miR-205 on PTEN expression was assessed in NSCLC cell lines with miR-205 mimics/inhibitor to elevate/ decrease miR-205 expression. Furthermore, the roles of miR-205 in regulating the biological properties of NSCLC cells, including growth, invasion and chemoresistance, were assayed using miR-205 mimic/inhibitor-transfected cells. The 3'-untranslated region (3'-UTR) of PTEN combined with miR-205 and this was confirmed by luciferase reporter assay and western blotting. miR-205 expression was increased in NSCLC cell lines as well as in tissues. Overexpression of miR-205 promoted growth, migration and invasion, and enhanced the chemoresistance of NSCLC cells. Luciferase activity and western blotting demonstrated that miR-205 negatively regulated PTEN at a posttranscriptional level. However, miR-205 knockdown suppressed these processes in A549 cells and increased the expression of PTEN protein. Furthermore, overexpression of PTEN exhibited effects identical with those of the miR-205 inhibitor in NSCLC cells. Our results demonstrated that miR-205 is involved in the tumorigenesis of NSCLC through modulation of the PTEN signaling pathway.
\end{abstract}

Correspondence to: Dr Yaping Huang, Department of Respiratory Medicine, Xiangyang Central Hospital, Xiangyang, Hubei 441021, P.R. China

E-mail: yapinghuang0710@163.com

Key words: non-small cell lung cancer, miR-205, invasion, chemotherapy

\section{Introduction}

Lung cancer is the leading cause of cancer-related mortality worldwide with only a $15 \%$ overall 5 -year patient survival rate (1). It is estimated that more than 1,600,000 new lung cancer cases are diagnosed each year. Non-small cell lung cancer (NSCLC) which includes adenocarcinomas, large cell carcinomas, and squamous cell carcinomas, contribute to $\sim 85 \%$ of the total lung cancer cases (2). Although recent advances have been made in clinical diagnosis and therapeutic treatment, the overall 5-year mortality rate has remained unfavorable since the 1970s (3). Further investigation of the underling mechanisms of NSCLC development and progression is thus important for improving the diagnosis, prevention and therapy of this disease.

microRNAs (miRNAs) are a class of endogenous noncoding RNAs which suppress gene expression through base pairing with the 3'-untranslated region (3'-UTR) of target mRNAs, leading to translational repression or mRNA decay (4). Emerging evidence has revealed that aberrant expression of miRNAs may induce alterations in a variety of biological processes, including proliferation, differentiation and migration. Numerous deregulated miRNAs, such as miR-21, miR-29b, miR-100, miR-155, miR-138, miR-449c and miR-847, have been shown to regulate NSCLC cell proliferation, migration and/or invasion (5-10). These findings indicate that dysfunctions of miRNAs may be associated with NSCLC. Recent studies have identified a series of miRNAs that are deregulated in NSCLC, including miR-205 (11). It was shown that ectopic expression of miR-205 in endometrial cancer promoted tumor proliferation and invasion (12). Xie et al reported that miR-205 overexpression increased cell proliferation and promoted migration of human cervical cancer cells (13). These data suggest a potential tumor promotive function of miR-205. However, the role and the molecular mechanisms of miR-205 in NSCLC remain poorly understood.

In the present study, we confirmed that upregulation of miR-205 was frequently found in NSCLC cell lines and tissue samples. Furthermore, downregulation of miR-205 by an miR-205 inhibitor markedly inhibited the migration, invasion and reversed the chemoresistance of NSCLC cells partially through targeting the tumor-suppressor gene, phosphatase and tensin homolog (PTEN). Our findings suggest a new therapeutic strategy for NSCLC. 


\section{Materials and methods}

Cell culture and tissue samples. NSCLC cell lines A549, SPC-A1, SK-MES-1, and the normal human bronchial epithelial cell line 16HBE were obtained from the Institute of Biochemistry and Cell Biology (Shanghai, China) and were maintained in RPMI-1640 containing 10\% fetal bovine serum, $100 \mu \mathrm{g} / \mathrm{ml}$ streptomycin, and $100 \mathrm{IU} / \mathrm{ml}$ penicillin at $37^{\circ} \mathrm{C}$ with $5 \% \mathrm{CO}_{2}$. Twenty-eight human NSCLC tissue samples and corresponding non-tumor tissues were collected from Xiangyang Central Hospital. Tissues were snap-frozen in liquid nitrogen immediately after surgery and stored at $80^{\circ} \mathrm{C}$. Informed consent was obtained from all patients prior to surgery. This study was approved by the Xiangyang Central Hospital Ethics Committee.

Constructs and transfection. miR-205 mimics (catalog\# miR10000266-1-2), miR-205 inhibitor (catalog\# miR10000266-1-2) and the corresponding controls were purchased from RiboBio Co., Ltd. (Guangzhou, China). For the luciferase activity assay, the wild-type (WT) or mutated (Mut) human PTEN 3'-UTR sequences containing the potential binding sites (nucleotides 759-765 of PTEN 3'-UTR) were amplified and inserted into the psiCHECK-2 vector (Promega, Beijing, China) between the XhoI and NotI restriction sites using the following primers: sense, 5'-CTCGAGCCGCTGTC ACTGCTTGT-3' and antisense, 5'-GCGGCCGCAGGCAGCA CATGAAGCA-3'. Mutation was performed using a fast mutation kit from Agilent (Santa Clara, CA, USA). The human PTEN overexpression construct was generated by amplifying the PTEN sequences using the following primers: sense, 5'-GGATTCCCACAGGCTCCCAGACA-3' and antisense, 5'-GAATTCTTGCCACAAGTGCAAAGG-3', and were inserted into pcDNA3.0 between the BamHI and EcoRI restriction sites. PTEN shRNA was designed and generated by GeneChem (Shanghi, China).

The transfection was performed using Lipofectamine 2000 (Invitrogen, Calsbad, CA, USA) according to the manufacturer's instructions. The transfected cells were cultured in regular culture medium for 48-72 $\mathrm{h}$ before analysis.

Quantitative real-time PCR. RNA containing small RNA was isolated from the cell lines with a microRNA extraction kit and reverse transcribed using a reverse transcription kit (Tiangen, Beijing, China), and subjected to SYBR Green realtime PCR (Takara, Dalian, China). Reactions were performed using StepOnePlus instrument in triplicate. Primers for miR-205 and U6 were purchased from GeneCopoeia, Inc. (Rockville, MD, USA). Expression level of miR-205 was normalized to U6. Total RNA was extracted with TRIzol (Invitrogen) and reversely transcribed into cDNA using the reverse transcription kit. Expression of PTEN was normalized with GAPDH. PTEN primers included sense, 5'-CCA GGACCAGAGGAAACCT-3' and antisense, 5'-GCTAGC CTCTGGATTTGA-3'. GAPDH primers were sense, 5'-CAT CTTCTTTTGCGTCGCC-3' and antisense, 5'-AAAAGC AGCCCTGGTGAC-3'.

MTT assay. Cell viability was examined by MTT assay. Briefly, the transfected cells were plated into 96-well plates. After $12 \mathrm{~h}$ of incubation, cells were treated with different concentrations of chemotherapeutic drugs. Following $24 \mathrm{~h}$ of incubation, cell growth was determined after addition of $0.5 \mathrm{mg} / \mathrm{ml}$ MTT solution (Sigma, USA). After $4 \mathrm{~h}$, the culture medium was replaced with $100 \mu \mathrm{l}$ DMSO and vortexed for $10 \mathrm{~min}$. Absorbance at $490 \mathrm{~nm}$ was recorded using a microplate reader (Bio-Rad, USA).

Flow cytometric analysis of apoptosis. The level of apoptosis was determined by flow cytometry. Cells were collected, washed with cold PBS twice, fixed in ice cold $70 \%$ ethanol. Cells were then stained with FITC-Annexin V and propidium iodide (PI), and analyzed by Cell Quest software (Becton Dickinson, Franklin Lakes, NJ, USA). The percentage of apoptotic cells was calculated.

In vitro migration and invasion assays. For in vitro migration assays, $24 \mathrm{~h}$ after transfection, cells in serum-free medium were seeded into the upper chamber of an insert $(8-\mu \mathrm{m}$ pore size). For in vitro invasion assays, cells in serum-free media were seeded into the upper chamber coated with Matrigel (Sigma). Medium containing 10\% FBS was added to the lower chamber. After $24 \mathrm{~h}$ of incubation, cells remaining on the upper chamber were removed with scraping, whereas cells that migrated or invaded into the lower chamber were stained with $0.05 \%$ crystal violet, imaged and counted under a microscope (Olympus, Tokyo, Japan).

Luciferase assay. A549 cells were co-transfected with a mixture including $0.02 \mu \mathrm{g}$ of wild-type or mutated PTEN 3'-UTR and $0.08 \mu \mathrm{g}$ of miR205 mimic. Renilla luciferase or the control mimic was used as the negative control. Luciferase activities were analyzed using the Dual-Luciferase reporter assay system (Promega, Madison, WI, USA).

Statistical analysis. Statistical analysis was performed using SPSS 16.0 software. Data are presented as means \pm SD. Statistical analyses were carried out by one-way ANOVA or the Student's t-test. $\mathrm{P}<0.05$ was considered to indicate a statistically significant result.

\section{Results}

miR-205 is increased in the NSCLC cell lines and tissue samples. To investigate the biological role of miR-205 in NSCLC, quantitative real-time PCR was initially performed to measure miR-205 expression levels in three NSCLC cell lines and 28 NSCLC tissue samples. As shown in Fig. 1A, the expression of miR-205 in NSCLC cell lines was significantly increased when compared to that in the normal human bronchial epithelial cell line $(16 \mathrm{HBE})(\mathrm{P}<0.01)$. The expression of miR-205 was also substantially elevated in NSCLC tissues when compared to that in the corresponding non-tumor tissues $(\mathrm{P}<0.05$, Fig. 1B). These results suggest that the upregulation of miR-205 contributes to NSCLC carcinogenesis.

miR-205 promotes the growth of the NSCLC cell lines. To study the effect of miR-205 on the growth of NSCLC cells, we initially examined the effect of miR-205 mimic/inhibitor on miR-205 expression. miR-205 mimics or the inhibitor was 
A

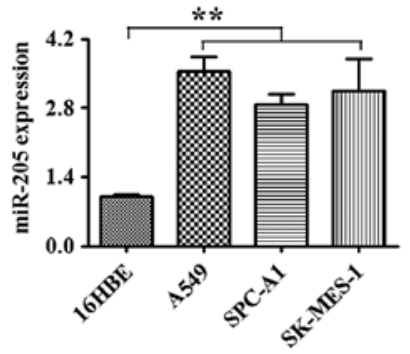

B

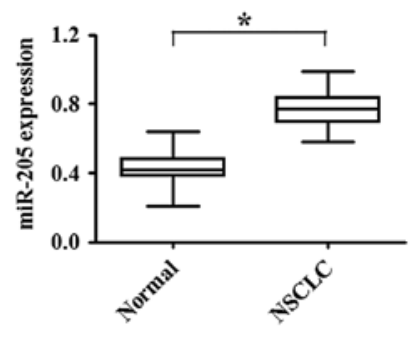

Figure 1. miR-205 expresssion is increased in NSCLC cell lines and tissue samples. (A) Expression of miR-205 was measured by quantitative real-time PCR in three NSCLC cell lines and 16HBE cells and (B) in 28 paired NSCLC and adjacent normal tissues. The relative expression level was normalized to U6. All experiments were performed in triplicate. ${ }^{*} \mathrm{P}<0.05,{ }^{* *} \mathrm{P}<0.01 \mathrm{com}-$ pared with the control.

A

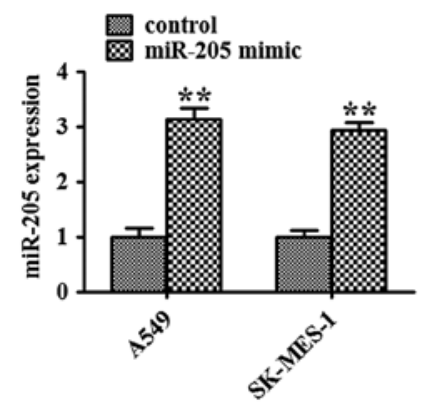

B

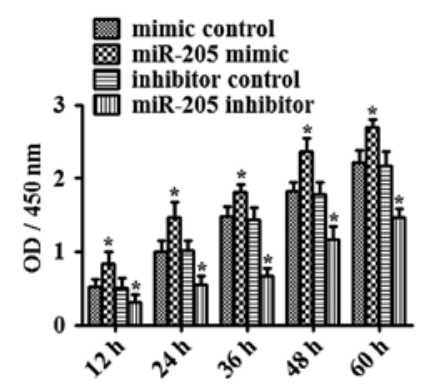

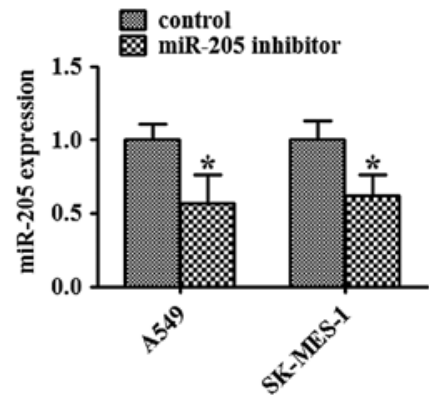

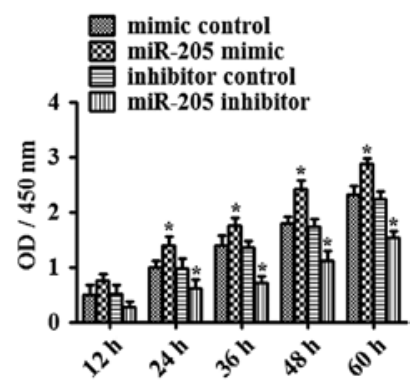

Figure 2. miR-205 promotes the growth of NSCLC cell lines. (A) Quantitative real-time PCR detection of miR-205 expression in NSCLC cells (A549 and SK-MES-1) at $48 \mathrm{~h}$ after transfection with miR-205 mimics or inhibitor. (B) MTT analysis of NSCLC cell (A549 and SK-MES-1) viability. All experiments were performed in triplicate. ${ }^{*} \mathrm{P}<0.05,{ }^{* *} \mathrm{P}<0.01$ compared with the control.

transiently transfected into A549 and SK-MES-1 cells. After $48 \mathrm{~h}$ of transfection, expression of miR-205 was determined by quantitative real-time PCR (Fig. 2A). Compared with the mimic control-transfected A549 or SK-MES-1 cells, expression of miR-205 in the miR-205 mimic-transfected cells was markedly increased $(\mathrm{P}<0.01)$. Compared with the inhibitor control-transfected A549 or SK-MES-1 cells, expression of miR-205 in the miR-205 inhibitor-transfected cells was significantly decreased $(\mathrm{P}<0.05)$. Next, MTT assay was used to examine the effect of miR-205 on the growth of NSCLC cells. As shown in Fig. 2B, miR-205 mimic-mediated elevation of miR-205 significantly promoted the growth of NSCLC cells,

A
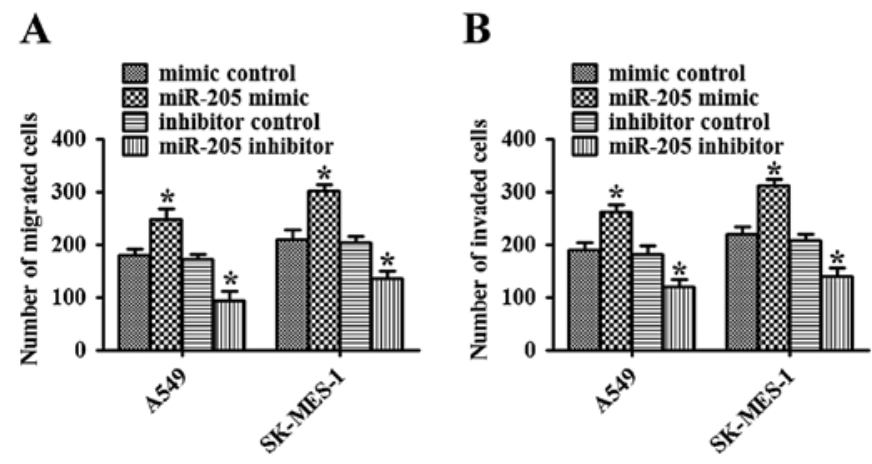

Figure 3. miR-205 promotes the migration and invasion of NSCLC cell lines. A549 and SK-MES-1 cells were transfected with the miR-205 mimic/ inhibitor, and the corresponding control and incubated for $24 \mathrm{~h}$. Transwell assays were performed to investigate the (A) migration and (B) invasion of NSCLC cells. All experiments were performed in triplicate. ${ }^{*} \mathrm{P}<0.05 \mathrm{com}-$ pared with the control.
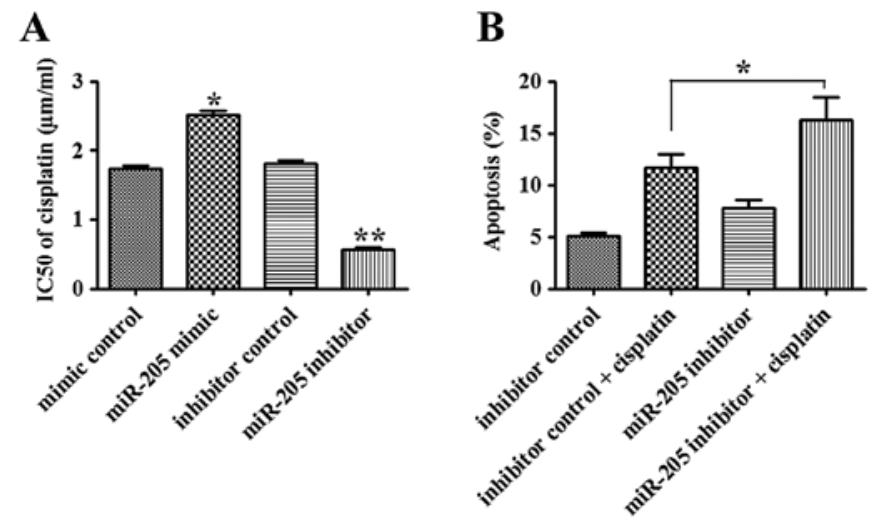

Figure 4. miR-205 promotes the chemoresistance of NSCLC cells (A) Alteration of $\mathrm{IC}_{50}$ values (cisplatin) in A549 cells transfected with miR-205 mimics/miR-205 inhibitor, and the corresponding control was analyzed by MTT. (B) Flow cytometric analysis of apoptosis in the miR205 inhibitor or the inhibitor control-transfected A549 cells combined with cisplatin $(1.50 \mu \mathrm{g} / \mathrm{ml})$ treatment. All experiments were performed at least in triplicate. ${ }^{*} \mathrm{P}<0.05,{ }^{* *} \mathrm{P}<0.01$ compared with the control.

while miR-205 inhibitor-mediated suppression of miR-205 substantially inhibited the growth of NSCLC cells $(\mathrm{P}<0.05)$. These data imply that miR-205 acts as an oncogene in human NSCLC.

miR-205 promotes the migration and invasion of the NSCLC cell lines. Cell invasion is an important aspect of cancer progression, and involves the migration and dissolution of extracellular matrix proteins of cancer cells into contiguous tissues. To investigate whether miR-205 has a direct functional role in promoting NSCLC cell migration and invasion, we assessed cancer cell invasion by Matrigel and migration through a transwell. As shown in Fig. 3A, overexpression of miR-205 promoted the migration of NSCLC cells, while inhibition of miR-205 significantly impeded the migration of NSCLC cells $(\mathrm{P}<0.05)$. Consistent with the results of the migration assay, upregulation of miR-205 promoted invasion, while downregulation of miR-205 inhibited invasion of NSCLC cells $(\mathrm{P}<0.05$, Fig. 3B). Therefore, these data suggest that miR-205 facilitates both migration and invasion of NSCLC cells. 
A

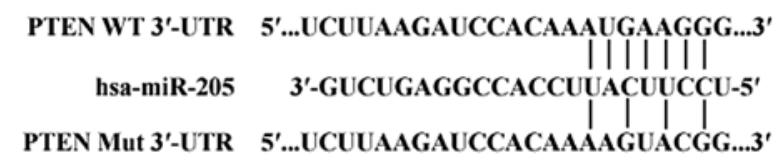

B

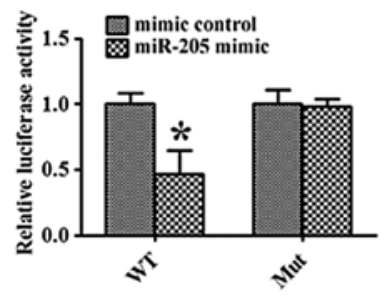

C

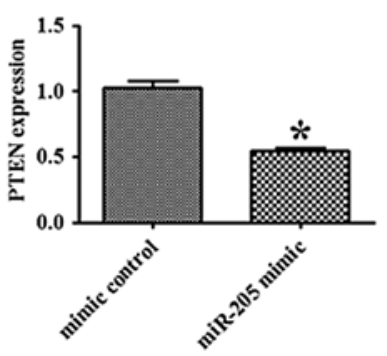

D
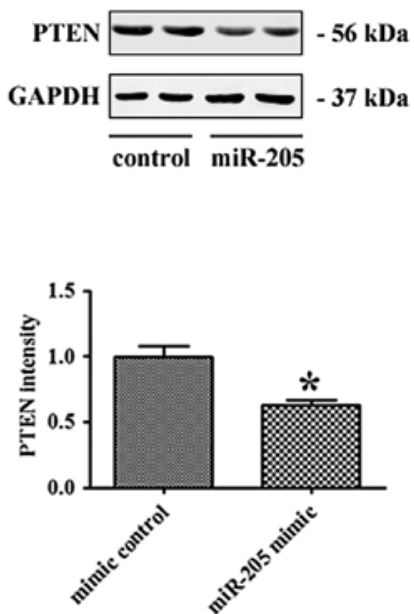

Figure 5. PTEN is a direct target of miR-205. (A) The wild-type and the corresponding mutants of the potential target sequences within the PTEN 3'-UTR for miR-205 are shown. (B) A549 cells were co-transfected with miR-205 mimics/control, and PTEN-WT-3'-UTR/PTEN-Mut-3'-UTR and incubated for $48 \mathrm{~h}$ and dual luciferase assays were performed. (C) Quantitative real-time PCR detection of PTEN expression in A549 cells transfected with the miR-205 mimic. (D) Western blot analysis of PTEN expression in A549 cells transfected with the miR-205 mimic. All experiments were performed at least in triplicate. ${ }^{*} \mathrm{P}<0.05$ compared with the control.

A

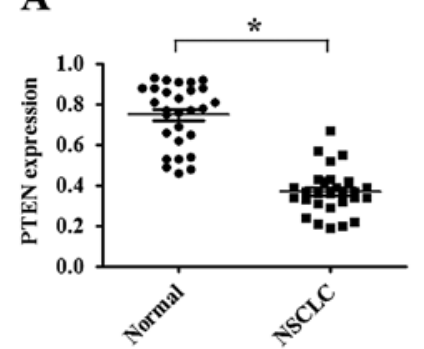

B

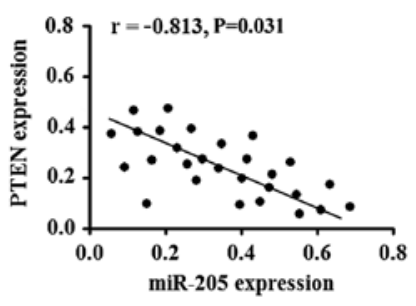

Figure 6. miR-205 is inversely correlated with PTEN expression. (A) Quantitative real-time PCR detection of PTEN expression in NSCLC tissues and that in the corresponding non-tumor tissues. (B) Correlation analysis between miR-205 and PTEN mRNA level in NSCLC tissues (Spearman's correlation analysis, $\mathrm{r}=-0.813 ; \mathrm{P}<0.05) .{ }^{*} \mathrm{P}<0.05$ compared with the control.

miR-205 promotes the chemoresistance of NSCLC cells. The effect of miR-205 on the sensitivity of NSCLC cells to chemotherapeutic agent, cisplatin, was investigated. Overexpression of miR-205 led to a significant increase in the $\mathrm{IC}_{50}$ value of cisplatin $(1.50 \mu \mathrm{g} / \mathrm{ml})$ in A549 cells when compared with that in the control group $(\mathrm{P}<0.05)$. In contrast, inhibition of miR-205 decreased the $\mathrm{IC}_{50}$ value of cisplatin in the A549 cells when compared with that in the control group $(\mathrm{P}<0.01$, Fig. 4A). When combined with cisplatin treatment, the apoptosis of A549 cells transfected with the miR-205 inhibitor was markedly enhanced when compared with that in the inhibitor control-transfected cells $(\mathrm{P}<0.05$; Fig. 4B). Our data suggest that miR-205 promotes chemoresistance of NSCLC cells via inhibition of apoptosis.

PTEN is a direct target of $m i R-205$. To explore the molecular mechanism of miR-205, we adopted bioinformatic algorithm, TargetScan 6.2, to predict many potential miR-205 target genes. Among them, PTEN was found to have a putative miR-205 binding site within its 3'-UTR. To verify whether

PTEN is a direct target of miR-205, WT or Mut 3'-UTR of PTEN was inserted into the downstream of the firefly luciferase gene (Fig. 5A). Then, miR-205 mimic or the mimic control-transfected A549 cells were co-transfected with WT or Mut 3'-UTR plasmids. As shown in Fig. 5B, miR-205 mimic decreased WT 3'-UTR luciferase activity $(\mathrm{P}<0.05)$, while it had no significant effect on Mut 3'-UTR luciferase activity. In addition, quantitative real-time PCR and western blot analyses showed that miR-205 mimic significantly decreased PTEN mRNA and protein levels in A549 cells ( $\mathrm{P}<0.05$; Fig. 5C and D). These results indicate that PTEN is a direct target of miR-205 in NSCLC cells.

miR-205 is inversely correlated with PTEN expression. Quantitative real-time PCR was performed to detect the expression of PTEN in 28 NSCLC tissue samples and the corresponding normal tissues. Data showed that the average level of PTEN mRNA was significantly decreased in NSCLC tissues when compared with that in the corresponding normal tissues ( $\mathrm{P}<0.05$; Fig. 6A). Moreover, we correlated PTEN with miR-205 expression in the same NSCLC tissues and found that PTEN mRNA level was inversely correlated with miR-205 level ( $\mathrm{P}<0.05$; Fig. 6B).

\section{Discussion}

Recently, numerous studies have focused on investigating the aberrant expression of miRNAs and their potential roles in tumor development and malignant transformation (14). miRNA expression profiles appear to be essential in cancer diagnosis, and strategies to modulate miRNA expression and function are considered to offer new opportunities for cancer therapy (15). In the present study, we demonstrated that overexpression of miR-205 promoted the growth, metastasis and chemoresistance of NSCLC cells partially by targeting PTEN, a tumor suppressor. 
miR-205 was reported to be elevated in many types of cancer including NSCLC $(11,16)$. It was found that miR-205 promoted tumor proliferation and invasion in endometrial cancer (12). Xie et al reported that miR-205 overexpression increased cell proliferation and promoted migration of human cervical cancer cells (13). In the present study, we initially demonstrated that the expression of miR-205 in NSCLC cell lines was much higher than that in a normal human bronchial epithelial cell line. Similarly, the expression of miR-205 was significantly elevated in NSCLC tissues when compared with that in the corresponding normal tissues. These data imply that the overexpression of miR-205 may play an essential role in NSCLC tumorigenesis. We found that inhibition of miR-205 significantly suppressed the growth of NSCLC cells. Herein, we also found that inhibition of miR-205 markedly inhibited the migration and invasion of NSCLC cells while overexpression of miR-205 promoted these capacities in NSCLC cells. Acquisition of resistance to chemotherapy or radiotherapy is a significant problem in cancer treatment, increasing the morbidity and mortality of patients. Thus, we next investigated whether miR-205 affects the chemosensitivity of NSCLC cells. Our data showed that inhibition of miR-205 significantly enhanced the sensitivity of NSCLC cells to the chemotherapeutic agent, cisplatin.

Wang et al found that miR-205 induced the radioresistance of human nasopharyngeal carcinoma by targeting the PTEN-Akt pathway (17). We also observed an inverse correlation between miR-205 and PTEN expression in NSCLC tissues. Several studies found that miR-205 acts as a tumor suppressor in several cancer types. For instance, Wu et al showed that ectopic expression of miR-205 suppressed proliferation and anchorage of breast cancer cell lines, MCF-7 and MDA-MB-231, by targeting vascular endothelial growth factor A (18). Boll et al found that miR-130a, miR-203 and miR-205 jointly impeded the growth of prostate cancer cells by induction of apoptosis and cell cycle arrest (19). These observations of increased or decreased miR-205 expression in different cancer types suggest that miR-205 may have different functions in different cancer types depending on the specific tumor context and target genes accompanied by other miRNAs deregulated as well (20).

PTEN, first identified as a tumor suppressor, is an important regulator of proliferation, differentiation and apoptosis (21). PTEN has been reported to be decreased in a wide variety of cancers including NSCLC $(22,23)$. Qu et al (24) reported that miR-205 contributed to the radioresistance of human nasopharyngeal carcinoma by targeting PTEN. Moreover, Karaayvaz et al (25) observed that miR-205 was negatively correlated with PTEN expression in endometrial cancer, and may act as a prognostic biomarker of endometrial cancer. To the best of our knowledge, there is no report concerning the associations of miR-205 and PTEN with the phenotypes of NSCLC cells, particularly chemoresistance. In the present study, we demonstrated that overexpression of miR-205 decreases both the mRNA and protein levels of PTEN in NSCLC cells, consistent with previous studies in other cancer types. These data suggest that miR-205 acts as an oncogene by suppressing PTEN expression in NSCLC.

In conclusion, the present study demonstrated the biological functions of miR-205, and its ability to promote growth, migration, invasion and chemoresistance of NSCLC cells by targeting PTEN. This finding suggests that inhibition of miR-205 may be a useful therapeutic strategy for NSCLC treatment.

\section{Acknowledgements}

This work was supported by the Natural Science Foundation of Hubei Province of China (grant no. 2011CDA065).

\section{References}

1. Travis WD, Brambilla E, Noguchi M, et al: International Association for the Study of Lung Cancer/American Thoracic Society/European Respiratory Society international multidisciplinary classification of lung adenocarcinoma. J Thorac Oncol 6: 244-285, 2011.

2. Jemal A, Ma J, Rosenberg PS, Siegel R and Anderson WF: Increasing lung cancer death rates among young women in southern and midwestern States. J Clin Oncol 30: 2739-2744, 2012.

3. Marshall E: Cancer research and the $\$ 90$ billion metaphor. Science 331: 1540-1541, 2011.

4. Boeri M, Pastorino U and Sozzi G: Role of microRNAs in lung cancer: microRNA signatures in cancer prognosis. Cancer J 18: 268-274, 2012.

5. Wu Y, Crawford M, Mao Y, et al: Therapeutic delivery of microRNA-29b by cationic lipoplexes for lung cancer. Mol Ther Nucleic Acids 2: e84, 2013.

6. Kesanakurti D, Maddirela DR, Chittivelu S, Rao JS and Chetty C: Suppression of tumor cell invasiveness and in vivo tumor growth by microRNA-874 in non-small cell lung cancer. Biochem Biophys Res Commun 434: 627-633, 2013.

7. Miao LJ, Huang SF, Sun ZT, et al: MiR-449c targets c-Myc and inhibits NSCLC cell progression. FEBS Lett 587: 1359-1365, 2013.

8. Zhang H, Zhao M, Lv Z, et al: MiR-138 inhibits tumor growth through repression of EZH2 in non-small cell lung cancer. Cell Physiol Biochem 31: 56-65, 2013.

9. Liu J, Lu KH, Liu ZL, Sun M, De W and Wang ZX: MicroRNA100 is a potential molecular marker of non-small cell lung cancer and functions as a tumor suppressor by targeting polo-like kinase 1. BMC Cancer 12: 519, 2012.

10. Yang M, Shen H, Qiu C, et al: High expression of miR-21 and miR-155 predicts recurrence and unfavourable survival in non-small cell lung cancer. Eur J Cancer 49: 604-615, 2013.

11. Mallick R, Patnaik SK and Yendamuri S: MicroRNAs and lung cancer: biology and applications in diagnosis and prognosis. J Carcinog 9: 2010. doi: 10.4103/1477-3163.67074.

12. Su N, Qiu H, Chen Y, Yang T, Yan Q and Wan X: miR-205 promotes tumor proliferation and invasion through targeting ESRRG in endometrial carcinoma. Oncol Rep 29: 2297-2302, 2013.

13. Xie H, Zhao Y, Caramuta S, Larsson C and Lui WO: miR-205 expression promotes cell proliferation and migration of human cervical cancer cells. PLoS One 7: e46990, 2012.

14. Du L and Pertsemlidis A: microRNA regulation of cell viability and drug sensitivity in lung cancer. Expert Opin Biol Ther 12: 1221-1239, 2012

15. Nana-Sinkam SP and Croce CM: Clinical applications for microRNAs in cancer. Clin Pharmacol Ther 93: 98-104, 2013.

16. Vosa U, Vooder T, Kolde R, Vilo J, Metspalu A and Annilo T: Meta-analysis of microRNA expression in lung cancer. Int $\mathbf{J}$ Cancer 132: 2884-2893, 2013.

17. Wang D, Wang S, Liu Q, Wang M, Wang C and Yang H: SZ-685C exhibits potent anticancer activity in both radiosensitive and radioresistant NPC cells through the miR-205-PTEN-Akt pathway. Oncol Rep 29: 2341-2347, 2013.

18. Wu H, Zhu S and Mo YY: Suppression of cell growth and invasion by miR-205 in breast cancer. Cell Res 19: 439-448, 2009.

19. Boll K, Reiche K, Kasack K, et al: MiR-130a, miR-203 and miR-205 jointly repress key oncogenic pathways and are downregulated in prostate carcinoma. Oncogene 32: 277-285, 2013.

20. Qin AY, Zhang XW, Liu L, et al: MiR-205 in cancer: an angel or a devil? Eur J Cell Biol 92: 54-60, 2013. 
21. Yamada KM and Araki M: Tumor suppressor PTEN: modulator of cell signaling, growth, migration and apoptosis. J Cell Sci 114: 2375-2382, 2001.

22. Panagiotou I, Tsiambas E, Lazaris AC, et al: PTEN expression in non small cell lung carcinoma based on digitized image analysis. J BUON 17: 719-723, 2012.

23. Li G, Zhao J, Peng X, Liang J, Deng X and Chen Y: The mechanism involved in the loss of PTEN expression in NSCLC tumor cells. Biochem Biophys Res Commun 418: $547-552,2012$.
24. Qu C, Liang Z, Huang J, et al: MiR-205 determines the radioresistance of human nasopharyngeal carcinoma by directly targeting PTEN. Cell Cycle 11: 785-796, 2012.

25. Karaayvaz M, Zhang C, Liang S, Shroyer KR and Ju J: Prognostic significance of miR-205 in endometrial cancer. PLoS One 7: e35158, 2012. 